\title{
Diretrizes para o Implante de Cardioversor Desfibrilador Implantável
}

\author{
José Carlos S. Andrade, Vicente Á vila N eto, Domingo M. Braile, Paulo R. S. Brofman, Alvaro R. B. Costa, \\ Roberto Costa, Silas S. Galvão Fo, Paulo R. A. Gauch, Fernando A. Lucchese, Martino Martinelli Fo, \\ Paulo T. J. Medeiros, José C. P. Mateos, João Pimenta, Roberto T. Takeda
}

São Paulo, SP

A morte súbita, responsável por cerca de $20 \%$ da mortalidade natural, continua sendo um dos maiores problemas da cardiologia. $O$ arsenal terapêutico utilizado no seu combate inclui os medicamentos antiarrítmicos, a ressecção cirúrgica, a ablação endocárdica por cateter e o implante de dispositivos elétricos: os cardioversores-desfibriladores implantáveis.

Sua utilização experimentou um enorme incremento nos últimos anos e vários megaestudos têm sido realizados para delinear a melhor opção terapêutica.

Os resultados desses estudos tornam mandatória a revisão do "Consenso para Implante de Cardioversor-Desfibrilador Implantável - Deca 1995".

Apesar da sua maior difusão e da aparente simplicidade de seu uso, as drogas antiarrítmicas, muitas vezes têm tido até mesmo o seu valor antiarrítmico contestado, em decorrência de seus efeitos pró-arrítmicos. No estudo CASH (Cardiac Arrest Study-Hamburg), iniciado em 1987 e concluído em 1993, que comparou o uso do desfibrilador implantável com vários fármacos, o estudo no subgrupo que utilizava propafenona foi interrompido, dada a mortalidade excessiva. Por outro lado, alguns fármacos, apesar da efetividade no controle de taquiarritmias, apresentam efeitos colaterais significativos, que motivaram a sua suspensão em $27 \%, 40 \%$ e $42 \%$ dos pacientes, respectivamente, dos grupos dos estudo CHF - STAD (Veterans Affairs Survival Trial of Antiarrhythmic Therapy in Congestive Heart Failure), EMIAT (European Amiodarone Myocardial Infarction - Trial) e CAMIAT (Canadian Amiodarone Myocardial Infarction Trial).

Além do efeito pró-arrítmico, o insucesso da terapêutica medicamentosa pode ocorrer devido à escolha incorreta do fármaco, à dosagem inadequada ou ao uso irregular por parte do paciente e ultimamente tem sido contestado o seu valor nos pacientes com comprometimento importante da função ventricular.

Consenso do Departamento de Estimulação Cardíaca Artificial da Sociedade Brasileira de Cirurgia Cardiovascular, 1999

Correspondência: Paulo R. A. Gauch - DECA - Rua Beira Rio, 45 - $7^{\circ}$ - conj 73 - 04548-050 - São Paulo, SP
Os cardioversores-desfibriladores implantáveis têm se mostrado a alternativa terapêutica mais eficiente para interromper taquicardias ventriculares sustentadas e fibrilações ventriculares, sendo responsáveis por uma redução expressiva na incidência de morte súbita, evidenciada nos estudos CASH, MADIT (Multicenter Automatic Desfibrillator Implantation Trial) e AVID (Antiarrythmicos Versus Implantable Desfibrillator). Este último, projetado para 1.200 pacientes também foi interrompido em virtude das taxas de mortalidade muito discrepantes entre seus diferentes subgrupos, o que levou o comitê dirigente a considerar anti-ética a continuidade do grupo de pacientes que recebia tratamento apenas com fármacos.

No MADIT, os pacientes com infarto agudo do miocárdio prévio e disfunção ventricular (fração de ejeção menor ou igual a $35 \%$ ), que apresentavam taquicardias ventriculares sustentadas ou fibrilação ventricular induzidas no estudo eletrofisiológico, foram divididos em dois grupos: um que recebeu o implante de cardioversor-desfibrilador implantável e outro, terapia antiarrítmica convencional. A discrepância da mortalidade após seguimento médio de 27 meses (15\% e $38 \%$ respectivamente) fez com que o Food and Drug Administration (FDA) dos Estados Unidos da América autorizasse a indicação de cardioversor-desfibrilador implantável para tais pacientes.

Recentemente, em abril de 1998, o American College of Cardiology (ACC) e a American Heart Association (AHA) revisaram as indicações para o implante dos cardioversor-desfibrilador implantável num "Executive Summary - ACC/AHA Practice Guidelines".

Os vários estudos realizados evidenciaram a alta efetividade do cardioversor-desfibrilador implantável na interrupção de taquiarritmias ventriculares letais e a expressiva redução de mortalidade com o seu uso, mudando a imagem terapêutica do mesmo, que hoje chega a ser considerado a opção inicial de tratamento em muitos casos. Para isso muito contribuiu a evolução tecnológica do cardioversor-desfibrilador implantável, com redução do seu tamanho e estimulação bicameral, possibilitando o seu implante em uma loja peitoral, uma técnica menos cruenta, mais estética e mais econômica. Ademais, a leitura eletrocardiográfica em átrio e em ventrículo propicia, pela discriminação das arritmias 
atriais e ventriculares, um diagnóstico mais preciso e um tratamento mais adequado.

Por todos esses motivos, e a despeito de sua atualidade, prevê-se que as indicações abaixo descritas seguramente continuarão a evoluir.

\section{Indicações}

Classe I-1) Sobreviventes de parada cardíaca por fibrilação ventricular/taquicardia ventricular conseqüente a causas irreversíveis e não transitória; 2) taquicardia ventricular sustentada espontânea, mal tolerada, sem alternativa terapêutica eficaz; 3 ) síncope de origem indeterminada, com indução no estudo eletrofisiológico de fibrilação ventricular ou taquicardia ventricular sustentada com comprometimento hemodinâmico significativo, quando a terapia farmacológica é ineficaz, mal tolerada ou inconveniente; 4) taquicardia ventricular não sustentada, em portador de infarto do miocárdio prévio e com importante disfunção de ventrículo esquerdo, com indução ao estudo eletrofisiológico de fibrilação ventricular ou taquicardia ventricular sustentadas e não suprimidas por drogas antiarrítmicas do grupo I.

Classe II - 1) Fibrilação ventricular/taquicardia ventricular espontânea, sustentada, em candidatos a transplante cardíaco; 2) condições familiares ou hereditárias de alto risco para taquiarritmias ventriculares letais, tais como a síndrome do QT longo; 3) taquicardia ventricular não sustentada em portador de infarto do miocárdio prévio com importante disfunção de ventrículo esquerdo, fibrilação ventricular ou taquicardia ventricular sustentada induzidas ao estudo eletrofisiológico; 4) síncope recorrente de etiologia indeterminada, na presença de importante disfunção ventricular, e fibrilação ventricular ou taquicardia ventricular sustentada com comprometimento hemodinâmico significativo, induzidas durante estudo eletrofisiológico, excluídas outras causas de síncope; 5) fibrilação ventricular/taquicardia ventricular espontânea ou induzida por estudo eletrofisiológico em pacientes submetidos a cardiomioplastia, ventriculectomia esquerda parcial ou outras técnicas alternativas.

Classe III - 1) Síncope de origem indeterminada em pacientes sem taquiarritmias espontâneas ou induzidas; 2) taquicardia ventricular incessante; 3) fibrilação ventricular ou taquicardia ventricular resultante de arritmias passíveis de ablação cirúrgica ou por cateter Wolf-Parkinson-White, taquicardia ventricular da via de saída do ventrículo direito, taquicardia ventricular idiopática do ventrículo esquerdo, taquicardia ventricular fascicular ou taquicardia ventricular ramo a ramo); 4) fibrilação ventricular/taquicardia ventricular conseqüentes a distúrbio transitório ou reversível; 5) doença psiquiátrica passível de agravamento pelo implante.

\section{Artigos consultados}

\footnotetext{
Cairns JA, Connoly SJ, Roberts R. et al. Canadian Amiodarone Myocardial Infarction Arrhythmia Trial (CAMIAT): radionale and protocol CAMIAT Investigators. Am J Cardiol 1993; 72: 87-94.

Camm AL, Julian D, Janse G, et al. The European Myocardial Infarct Amiodarone Trial (EMIAT). EMIAT Investigators. Am J Cardiol 1993; 72: 95-8.

Consenso para o implante de marcapasso cardíaco permanente e desfibriladorcardioversor-implantável - 1995 (Deca/SBCCV). Reblampa 1995; 8: 4-11.

Doval HC, Nul DR, Grancelli HO, et al. Grupo de Estudios de la Sobrevida en la Insuficiencia Cardiaca en Argentina (GESICA). Randomised trial of low-dose amiodarona in severe congestive heard failure. Lancet 1994; 344: 493-8.

Dreifus LS, Fisch C, Griffin JC, Gillette PC, et al. Guidelines for implantation of cardiac pacemaker and antiarrhythmia devices. Circulation 1991; 84: 455-67.

Fenelon G, Stambler BS, Paola AAV. Em que os grandes "trials" modificaram o tratamento das arritmias ventriculares? Rev Soc Cardiol ESP 1998; 8: 197-208.

Gregoratos G, Chertlin MD, Corril A, et al. ACC/AHA Guidelines for implanta-
}

tion of cardiac pacemakers and antiarrhythmia devices. Executive summary. A report of the ACC/AHA Task Force on Practice Guidelines. Circulation 1998; 97 : 1325-35.

Mirowski M, Reid PR, Mower MM, et al. Termination of malignant ventricular tachyarrthmias with an implanted automatic defibrillator in human beings. NEngl J Med 1980; 303: 322-8.

Moss A, Hall J, Cannom D, et al. MADIT Investigators improved survival with an implanted defibrillator in patients with coronary artery disease at high risk of ventricular arrhythmias. N Engl J Med 1996; 335: 1933-40.

Siebels J, Kuck KH. Implantable cardioverter defibrillator compared with antiarrhythmic drug treatment in cardiac arrest survivors (The Cardiac Arrest Study Hamburg). Am Heart J 1994; 127: 1139-44.

The Antiarrhythmics Versus Implantable Defibrillators (AVID) Investigators. A comparison of antiarrhythmic drug therapy with implantable defibrillators in patients resuscitated from near-fatal ventricular arrhythmias. N Engl J Med 1997; 337: $1575-83$. 